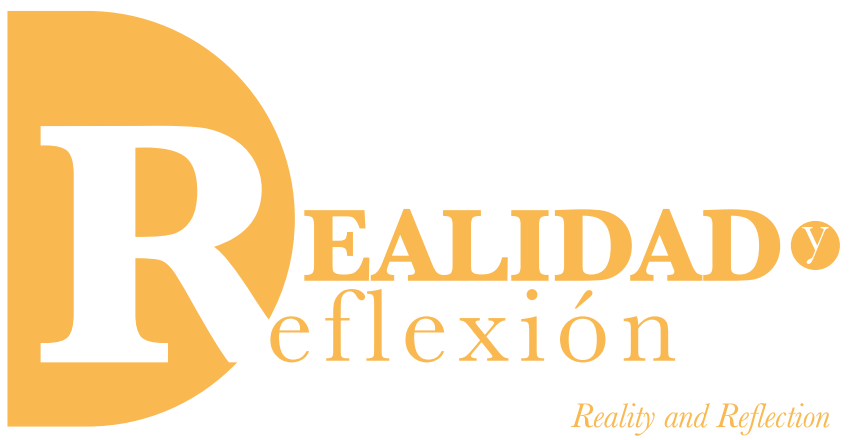

ISSN 1992-6510

Año 18, N 48, San Salvador, El Salvador, Centroamérica. Revista Semestral Julio-Diciembre 2018

YEAR 18, N 48, SAN SALVADOR, El SALVADOR, CENTRAL AMERICA. SEMESTRAL JouRNAL JULY-DECEMBER 2018

\title{
Competitividad global y educación. Una mirada al caso de El Salvador
}

\author{
Global competitiveness and education. \\ A look at the case of El Salvador
}

\author{
Rolando Balmore Pacheco \\ Profesor de Educación Básica, Ciudad Normal Alberto Masferrer. \\ Licenciado en Ciencias de la Educación, Universidad Francisco Gavidia. \\ Postítulo en Población y Desarrollo, Universidad de Chile. \\ Máster en Educación, Universidad de Louisville, Estados Unidos. \\ Estudiante de Doctorado en Gestión Pública y Ciencias Empresariales, \\ Instituto Centroamericano de Administración Pública, ICAP. \\ rpacheco@ufg.edu.sv \\ Fecha de recepción: 5 de septiembre de 2018 \\ Fecha aprobación: 17 de diciembre de 2018 \\ DOI: $10.5377 /$ ryr.v48i0.7089
}

\section{RESUMEN}

En las páginas siguientes se hace un análisis de dos de los principales indicadores empleados internacionalmente para medir el desempeño de las economías y la comparabilidad de las mismas entre los diferentes países, siendo estos el Índice de Competitividad Global (ICG) que es auspiciado por el Foro Económico Mundial y Doing Business, promovido por el Grupo Banco Mundial. El análisis se basa en la revisión -principalmente- de los reportes generados por ambas instituciones en los últimos tres períodos de medición, incluyendo el examen de los datos a nivel global, de región latinoamericana y de nivel nacional (caso El Salvador). Se incluye un análisis cualitativo de correlación entre los hallazgos de ambos indicadores y los resultados en educación. Finalmente se plantean algunas reflexiones finales, así como recomendaciones para la mejora en los sectores analizados principalmente desde las políticas públicas en educación.

Palabras clave: competitividad, El Salvador, educación, crecimiento.

\section{ABSTRACT}

In the following pages an analysis is made of two of the main indicators used internationally to measure the performance of economies and their comparability among different countries, these being the Global Competitiveness Index (GCI) that is sponsored by the Economic World Forum and Doing Business, promoted by the World Bank Group. The analysis is based on the review -mainly- on the reports generated by both institutions in the last three measured periods, including the examination of data at the global level, in the Latin American region and at the national level (El Salvador case). A qualitative analysis of the correlation between the findings of both indicators and the results in education is included. Finally, some reflections are proposed, as well as recommendations for improvement in the sectors analyzed mainly from public policies on education.

Keywords: competitiveness, El Salvador, education, growth. 


\section{Marco global de la competitividad}

El Foro Económico Mundial (WEF, por sus siglas en inglés), desde el año 1979, publica anualmente el Reporte de Competitividad Global, basado en el Índice de Competitividad Global (ICG), mediante el cual se analiza el desempeño de las economías de diversos países del mundo, estableciendo un ranking global con base en las puntuaciones de cada país. El análisis de competitividad se hace a partir de información que se recaba de cada país alrededor de 12 pilares, siendo estos: i) instituciones, ii) infraestructura, iii) estabilidad macroeconómica, iv) salud y educación básica, v) educación superior y capacitación, vi) eficiencia en el mercado de bienes, vii) eficiencia en el mercado laboral, viii) sofisticación del mercado financiero, ix) preparación tecnológica, $\mathrm{x}$ ) tamaño de mercado, xi) sofisticación empresarial e xi) innovación. Estos 12 pilares son desagregados en 107 variables para cuya medición se emplean datos duros y de percepción de los actores económicos y sociales. A su vez, los 12 pilares son agrupados en 3 subíndices: requerimientos básicos, potenciadores de eficiencia y sofisticación de la innovación.

De acuerdo con el reporte 2016-2017, los 8 países líderes con los mejores índices a nivel mundial son, en su orden: Suiza, Singapur, Estados Unidos, Holanda, Alemania, Suecia, Reino Unido y Japón. En el caso de América Latina es Chile el país mejor ubicado en el ranking para ese mismo período, colocándose en la posición 33, seguido de Panamá en la posición 42, México en la posición 51, Costa Rica en la posición 54, Colombia en la posición 61, Perú en la posición 69 y Uruguay en la posición 73. El Salvador obtuvo un score de 3.81 ubicándose en la posición 105, bajando 10 escalones con respecto al período 2015-2016, solo por arriba de Paraguay (posición 117), Bolivia (posición 121) y Venezuela (posición 130), en la región latinoamericana (Foro Económico Mundial, 2017).

El reporte del período 2017-2018, emitido recientemente por el WEF, confirma en términos generales las posiciones y tendencias observadas en períodos precedentes por los diferentes países a nivel global, con algunas leves variaciones, como es el caso de Hong Kong que entra en este período al top ocho de los países con mejor ranking en el Índice de Competitividad Global, desplazando a Japón de ese selecto grupo. A nivel de región, Chile es el país que continúa a la cabeza en América Latina, siempre en la posición 33 del ranking mundial. Es seguido por Costa Rica (posición 47), Panamá (posición 50), México (posición 51), Colombia (posición 66) y Perú (posición 72). El Salvador, con un score de 3.80 baja 4 peldaños con respecto al período anterior, ubicándose en la posición 109 del ranking mundial, solo por debajo de Paraguay (posición 112), Venezuela (posición 127) y Haití (posición 128) en la región latinoamericana (Foro Económico Mundial, 2018). Ver Tabla n. ${ }^{\circ} 1$.

Esta caída en el ICG revela una lamentable reversión del avance en competitividad de E1 Salvador, pues según los registros del WEF, es la posición más baja que el país ocupa desde el período 2007-2008, cayendo aún más bajo del lugar 101 que alcanzó en el reporte del período 2012-2013. En un análisis comparativo, a nivel global, el desempeño de competitividad de El Salvador es muy similar al de Mongolia, Bangladesh, República Kirguiza, Gabón, Senegal, Cabo Verde y Etiopía, mientras que a nivel latinoamericano es muy similar al de Argentina y Nicaragua. 
Tabla n. ${ }^{\circ} 1$

Indice Global de Competitividad 2017-2018

\begin{tabular}{c|c|c|c}
\hline Posición & Economía & Score & Posición previa \\
\hline 1 & Suiza & 5.86 & 1 \\
\hline 2 & Estados Unidos & 5.85 & 3 \\
\hline 3 & Singapur & 5.71 & 2 \\
\hline 4 & Holanda & 5.66 & 4 \\
\hline 5 & Alemania & 5.65 & 5 \\
\hline 6 & Hon Kong SAR & 5.53 & 9 \\
\hline 7 & Suecia & 5.52 & 6 \\
\hline 8 & Reino Unido & 5.51 & 7 \\
\hline 33 & Chile & 4.7 & 33 \\
\hline 47 & Costa Rica & 4.50 & 54 \\
\hline 50 & Panamá & 4.44 & 42 \\
\hline 51 & México & 4.44 & 51 \\
\hline 66 & Colombia & 4.29 & 67 \\
\hline 72 & Perú & 4.22 & 73 \\
\hline 76 & Uruguay & 4.15 & 81 \\
\hline 80 & Brasil & 4.14 & $\mathbf{1 0 5}$ \\
\hline 109 & El Salvador & 3.77 & \\
\hline
\end{tabular}

Fuente: Foro Económico Mundial, 2018.

El score global que como país obtuvo El Salvador en el reporte del 2016-2017 fue de 3.81 (posición 105 en el ranking global), en el subíndice de requerimientos básicos obtuvo un score de 4.15 (posición 100), en el subíndice potenciadores de eficiencia alcanzó un score de 3.67 (posición 101) y en el subíndice de sofisticación de la innovación registró un score de 3.16 (posición 117). Para el período 2017-2018, el país experimenta una baja de cuatro posiciones en el ranking global, con un score de 3.80, manteniendo puntajes muy similares en los 3 subíndices en que se desagrega el ICG.

Si bien los datos de país revelan retrocesos de competitividad en términos generales, la desagregación por subíndices pone al descubierto las deficiencias y factores de competitividad más críticos y a los cuales el país deberá prestarles especial atención. E1 Salvador enfrenta, de acuerdo con los reportes de los últimos nueve años, que es en los pilares sofisticación de empresas e innovación en donde se localizan los retos más importantes, pues en ambos ha registrado bajas significativas de manera sostenida, bajando en el primero de ellos 40 posiciones, con lo cual descendió al lugar 104; y en el segundo descendió 31 escalones, bajando al lugar 130 (Foro Económico Mundial, 2018). 
Tabla n. ${ }^{\circ} 2$

Indice Global de Competitividad de El Salvador, 2017-2018

(Desagregado por pilares de competitividad)

\begin{tabular}{|c|c|c|}
\hline Subíndice y Pilares & Score & Posición \\
\hline Competitividad global & 3.8 & 109 \\
\hline Subíndice A: Requerimientos básicos & 4.1 & 99 \\
\hline Instituciones & 2.7 & 133 \\
\hline Infraestructura & 4.0 & 77 \\
\hline Estabilidad macroeconómica & 4.5 & 85 \\
\hline Salud y educación primaria & 5.3 & 100 \\
\hline Subíndice B: Potenciadores de eficiencia & 3.6 & 107 \\
\hline Educación superior y capacitación & 3.5 & 104 \\
\hline Eficiencia el mercado de bienes & 4.0 & 109 \\
\hline Eficiencia del mercado laboral & 3.4 & 125 \\
\hline Sofisticación del mercado financiero & 4.2 & 57 \\
\hline Preparación tecnológica & 3.4 & 95 \\
\hline Tamaño de mercado & 3.3 & 91 \\
\hline Subíndice C: Sofisticación de empresas e innovación & 3.1 & 120 \\
\hline Sofisticación de empresas & 3.6 & 104 \\
\hline Innovación & 2.6 & 130 \\
\hline
\end{tabular}

Fuente: Foro Económico Mundial, 2018

La sofisticación de las empresas hace referencia a la calidad de las redes empresariales (cantidad y calidad de oferentes locales, así como su interacción) y a la calidad de las operaciones y estrategias de las empresas particulares. El WEF señala que "cuando las empresas están interconectadas, la eficiencia se ve reforzada, y hay mayor capacidad para la innovación en procesos y creación de productos" (Foro Económico Mundial, 2017). Sin duda, este es un factor de competitividad al que desde las políticas públicas E1 Salvador deberá ponerle especial atención.

Por su parte, el pilar de innovación hace referencia a la incorporación de tecnología en los procesos de producción de bienes y servicios, la cual es clave para incrementar la productividad. En esta misma línea el WEF señala que la innovación tecnológica favorece además del mejoramiento de la productividad, el diseño de nuevos productos y servicios que permiten sostener altos salarios en las economías más avanzadas.

Si bien los requerimientos básicos como la infraestructura, la solidez institucional, la salud, la educación y la estabilidad macroeconómica son importantes para la mejora de la competitividad de las economías, lo cierto es que ello se vuelve insuficiente, pues los avances en términos de incorporación tecnológica, sofisticación 
empresarial e innovación han demostrado jugar un papel determinante a la hora de aumentar el PIB (Producto Interno Bruto) per cápita en los países que se colocan a la vanguardia en el ICG (Foro Económico Mundial, 2017).

\section{Una mirada a la competitividad desde los pilares del sector Educación}

Para el cálculo del ICG, el Foro Económico Mundial considera dos pilares relativos al sector Educación: salud-educación y educación superior-capacitación. Ambos pilares, si bien son analizados de manera independiente, debe decirse que se vuelven influyentes en el resto de factores y variables de competitividad de los países. Los sistemas educativos son los responsables de preparar a las personas con las habilidades requeridas para una participación exitosa en los ámbitos económico y social. Desde las políticas públicas en educación se puede hacer una contribución al resto de pilares que conforman la compleja trama de factores que le confieren competitividad a un país. La cantidad y la calidad de la educación (inicial, básica, media, técnica y superior) que recibe la población también es fundamental para el crecimiento y desarrollo económico de una nación. Congruente con esta línea de pensamiento el WEF sostiene que "los trabajadores sanos y educados estarán más preparados para ser más productivos que los que están enfermos y que no cuenten con una educación básica”, agrega además que "para tener una economía competitiva en el ámbito internacional, es necesario tener una fuerza laboral calificada y que ofrezca productos superiores en la cadena de valor" (Foro Económico Mundial, 2017).

\subsection{Salud-educación primaria}

De acuerdo con el reporte 2016-2017, los 8 países líderes en salud y educación primaria a nivel mundial son, en su orden: Finlandia con score de 6.89, Singapur con 6.75, Bélgica con 6.74, Holanda con 6.67, Japón con 6.64, Nueva Zelanda con 6.61, Islandia con 6.59 y Suiza con 6.58. En el caso de América Latina es Costa Rica el país mejor ubicado, que con un score de 6.21 ocupa la posición 35, seguido de Panamá en la posición 67, Ecuador en la posición 68, Chile en la posición 71 y México en la posición 74. El Salvador, para el período en mención, siempre en el pilar salud y educación primaria, se ubica en la posición 96 del ranking mundial, con un score de 5.37, solo por arriba de Brasil (posición 99), Bolivia (posición 101) y Paraguay (posición 106).

Los resultados del ICG son concordantes con los hallazgos del Programa para la Evaluación Internacional de Alumnos (Programme for International Student Assessment - PISA), que es auspiciado por la OCDE, siendo su objetivo evaluar el aprendizaje de los alumnos cuando llegan al final de la etapa de enseñanza obligatoria, es decir a los 15 años. El Programa PISA se pretende sea un recurso para generar $\mathrm{y}$ ofrecer información abundante $\mathrm{y}$ detallada que permita a los países miembros generar las políticas públicas necesarias para mejorar la calidad de sus sistemas educativos. Las pruebas PISA exploran las áreas de lectura, matemáticas y ciencias, siendo el énfasis de la evaluación, la medición del dominio de los procesos, la comprensión de los conceptos y la habilidad de actuar o funcionar en varias situaciones dentro de cada dominio (OCDE, 2012). Los países con mejor ranking en el ICG, en la mayoría de 
los casos, lo son también en el ranking PISA, lo que revela la alta correlación entre educación y competitividad de las economías. Los ocho países con mejor ranking en el ICG del período 2017-2018, se encuentran también entre los primeros 30 países con mejores resultados en las pruebas PISA del reporte de 2016. El ranking de PISA 2016 ubica a Singapur a la cabeza, seguido de Japón, Estonia, Taiwán, Finlandia, Macao, Canadá y Vietnam (OCDE, 2016).

Tabla n. ${ }^{\circ} 3$

Comparación de ranking IGC y ranking PISA, 2016
Ningún país de América Latina aparece en el top 30 del ranking de PISA 2016, siendo Argentina el país de la región mejor ubicado (posición 38), seguido de Chile (posición 44), Uruguay (posición 47), Costa Rica (posición 55), Colombia (posición 57) y México (posición 58). El Salvador no se encuentra en la lista de países que han tomado parte del Programa para la Evaluación Internacional de Alumnos (PISA), ni se proyecta sea incluido en la edición de 2018. Héctor Samour, viceministro de Educación de

\begin{tabular}{c|c|c}
\hline Economías & Ranking IGC 2018 & Ranking PISA 2016 \\
\hline Suiza & 1 & 18 \\
\hline Estados Unidos & 2 & 25 \\
\hline Singapur & 3 & 1 \\
\hline Holanda & 4 & 16 \\
\hline Alemania & 5 & 9 \\
\hline Hong Kong SAR & 6 & 28 \\
\hline Suecia & 7 & 15 \\
\hline Reino Unido & 8 & 2 \\
\hline Japón & 9 & 5 \\
\hline Finlandia & 10 & 4 \\
\hline Taiwán & 15 & 3 \\
\hline Estonia & 29 & 44 \\
\hline Chile & 33 & 55 \\
\hline Costa Rica & 47 & 58 \\
\hline México & 51 & 57 \\
\hline Colombia & 66 & 47 \\
\hline Uruguay & 76 & 38 \\
\hline Argentina & 92 & \\
\hline
\end{tabular}

Fuente: Foro Económico Mundial, 2018 y OCDE, 2016. 
El Salvador, al ser consultado en 2012 sobre por qué El Salvador continúa sin participar en las diferentes ediciones de PISA, manifestó que el país aún no cuenta con la calidad educativa para hacerlo, que hay que afianzar más el sistema educativo nacional, elevar más su calidad, para asegurar un buen suceso cuando se participe, agregando que esa participación sería lo más pronto posible, situación que aún no ocurre. Samour añadió que para mejorar la calidad educativa se debe invertir más en programas que la garanticen, es necesario que el gobierno central tome en cuenta que debe invertirse al menos el seis por ciento del Producto Interno Bruto en la educación (Periódico La Página, 2012).

Para el período 2008-2009, el WEF reportó a El Salvador en la posición 86 en el pilar de salud y educación primaria, solo por debajo de Costa Rica (posición 29) en Centro América. Si comparamos este dato con el correspondiente al período 2017-2018, se advierte que El Salvador ha descendido en catorce posiciones (posición 100), lo cual revela un importante retroceso en materia de salud y educación primaria, dejando en evidencia la ineficiencia de las políticas públicas que en estas dos áreas se han implementado en los últimos diez años.

Los datos del ICG correspondientes al país son concordantes con los resultados que los estudiantes salvadoreños obtienen en la Prueba de Aprendizajes y Aptitudes para los Egresados de Educación Media (conocida como PAES), los cuales -como promedio nacional- son sumamente bajos. En el 2009, el promedio nacional de la PAES fue de 4.99 (en una escala de 0 a 10), promedio que en los últimos ocho años se ha mantenido más o menos constante, con algunos altibajos. En el año 2017 el promedio nacional de la PAES fue 5.36, lo que desde luego no es satisfactorio y deja al descubierto importantes déficit formativos en la población salvadoreña, así como la inefectividad del sistema educativo nacional (MINED, 2017). Ver Tabla n. 4.

El año 2007 El Salvador tomó la decisión de participar en el Estudio Internacional de Tendencias en Matemáticas y Ciencias (TIMSS, por sus siglas en inglés), que tiene como propósito medir las tendencias en el rendimiento de los estudiantes de cuarto y octavo grados en matemáticas y ciencias. Ese año se evaluaron aproximadamente 425,000 estudiantes de 59 países y ocho entidades subnacionales. La aplicación cada cuatro años posibilita la obtención de información sobre el progreso relativo entre grados, puesto que los estudiantes de cuarto evaluados en un ciclo de TIMSS estarán cursando octavo en la siguiente cohorte. Esta es la primera vez que El Salvador participa en una prueba de esta magnitud internacional. En la región latinoamericana, sólo los países más desarrollados y con una amplia tradición en evaluación estandarizada como Brasil, México, Chile, Colombia y Uruguay han participado en alguno de los ciclos del estudio TIMSS.

En la asignatura de matemática, cuarto grado, E1 Salvador ocupó el lugar 33 entre los 37 países participantes. Una posición más baja que el promedio internacional, similar a la de Marruecos y Túnez y más alto que el de Qatar y Yemen. Sólo 19 países, todos asiáticos o europeos, obtuvieron promedios superiores al promedio internacional TIMSS. Los primeros lugares fueron ocupados por países asiáticos. 
Tabla n. ${ }^{\circ} 4$

Resultados de la PAES, 2009-2017

\begin{tabular}{|c|c|c|c|c|c|c|c|c|c|}
\hline Año & $\mathbf{2 0 0 9}$ & $\mathbf{2 0 1 0}$ & $\mathbf{2 0 1 1}$ & $\mathbf{2 0 1 2}$ & $\mathbf{2 0 1 3}$ & $\mathbf{2 0 1 4}$ & $\mathbf{2 0 1 5}$ & $\mathbf{2 0 1 6}$ & $\mathbf{2 0 1 7}$ \\
\hline Promedio nacional (1-10) & 4.99 & 5.14 & 4.85 & 5.0 & 5.3 & 5.2 & 5.3 & 5.26 & 5.36 \\
\hline
\end{tabular}

Fuente: MINED 2017.

Los dos países con el más alto rendimiento fueron Hong Kong SAR y Singapur, seguidos por China (Taiwán), Japón y Kazakhstan. Entre todos los países participantes, sólo Yemen y Marruecos tienen un Índice de Desarrollo Humano (IDH) más bajo que el de El Salvador. El IDH tiene una fuerte correlación positiva con el logro académico (entre más alto desarrollo humano mejor logro académico) y de acuerdo con él, El Salvador ocupa una posición adecuada o esperada. La posición ocupada por El Salvador, aunque es relativamente más baja que la de Colombia, el otro país latinoamericano participante en el estudio, es similar a la ocupada por el resto de países latinoamericanos que han participado previamente en los ciclos anteriores.

En el octavo grado, siempre en Matemática, E1 Salvador ocupó el lugar 45 entre los 48 países participantes sobre los que se reportan resultados. Una posición más baja que el promedio internacional, similar a la de Kuwait y Arabia Saudita y más alto que el de Gana y Qatar. Sólo 12 países, todos asiáticos o europeos, obtuvieron promedios superiores al promedio internacional TIMSS. Al igual que en $4^{\circ}$, los primeros lugares fueron ocupados por países asiáticos. Los países con el más alto rendimiento fueron, en su orden, China (Taiwán), Corea del Sur y Singapur, seguidos por Hong Kong SAR y
Japón. En este grado, El Salvador, tiene un logro académico por debajo de lo esperado según su IDH (TIMSS, 2007).

En la asignatura de Ciencias, Cuarto Grado, El Salvador ocupó el lugar 30 entre los 37 países participantes. Una posición más baja que el promedio internacional, similar a la de Colombia y más alto que el de Argelia, Kuwait, Túnez, Marruecos, Qatar y Yemen. Al igual que en Matemáticas, los primeros lugares fueron ocupados por países asiáticos. Los dos países con el más alto rendimiento fueron Singapur, China (Taiwán) y Hong Kong SAR, seguidos por, Japón y Rusia. El Salvador ocupa una posición adecuada o esperada en función de su IDH. En este grado, la posición ocupada por El Salvador es similar a la de Colombia, el otro país latinoamericano participante en el estudio, y también es similar a la ocupada por el resto de países latinoamericanos que han participado en los ciclos anteriores (TIMSS, 2007).

En el octavo grado, El Salvador ocupó el lugar 45 entre los 48 países participantes. Una posición más baja que el promedio internacional, similar a la de Arabia Saudita y más alto que el de Botswana, Qatar y Gana. Como en Cuarto Grado, los primeros lugares fueron ocupados por países asiáticos. Los países con el más alto rendimiento fueron Singapur, China (Taiwán) y 
Japón, seguidos por Corea del Sur e Inglaterra. De acuerdo con su IDH, El Salvador ocupa una posición adecuada o esperada (TIMSS, 2007).

La ineficiencia del sistema educativo se traduce en una fuerza laboral menos competente $y$ divorciada de las necesidades de la sociedad, lo que le impide adaptarse a los requerimientos de productividad y competitividad que demandan los entornos laborales en la actualidad. De allí, que el desafío del sistema educativo salvadoreño sea el preparar a su población con un rango de habilidades para la vida ciudadana y el desempeño en los diversos entornos productivos.

\subsection{Educación superior y capacitación}

El pilar de educación superior y capacitación que mide el ICG hace referencia, como ya se mencionó antes, a la calidad y cantidad de la educación superior, así como a la calidad y disponibilidad de la formación en el puesto de trabajo. Los países con más alto score y ranking en este pilar de competitividad, según el reporte del período 2016-2017, son en su orden: Singapur con un score de 6.29, Finlandia con 6.16, Holanda con 6.07, Suiza con 6.03, Bélgica con 5.99, Dinamarca con 5.95, Noruega con 5.94 y Estados Unidos con 5.91. En América Latina es Chile el país mejor ubicado, que con un score de 5.20 se ubica en la posición número 28, seguido de Costa Rica en la posición 35, Argentina en la posición 40, Uruguay en la posición 48 y Venezuela en la posición 53. El Salvador, para el período en mención, siempre en el pilar educación superior se ubica en la posición 103 , con un score de 3.57 , solo por arriba de Paraguay (posición 107) y Nicaragua (posición 116), esto en la región latinoamericana.
Para el período 2008-2009, el WEF reportó a El Salvador en la posición 95 en el pilar de educación superior y capacitación. Al comparar este dato con el correspondiente al período 2016-2017, se advierte que El Salvador ha descendido en ocho posiciones, lo cual revela un importante retroceso en materia de calidad de la educación superior, así como en formación para el trabajo, dejando también en este caso al descubierto las ineficiencias de las instituciones de educación superior del país y de las políticas públicas que en esta área se han implementado en la última década.

Estudios internacionales señalan que la calidad de un sistema educativo tiene como techo la calidad de sus docentes, y que la única manera de mejorar dichos sistemas es mejorando la calidad de cómo estos enseñan (M. Barber y M. Mourshed, 2008). Sin embargo, a pesar de la importancia de los docentes en la promoción de los aprendizajes, en la mayoría de los países de esta región -incluido El Salvador- los sistemas de reclutamiento, formación, retención y apoyo de maestros son todavía inadecuados. Además, los grupos de interés que más deberían involucrarse con los gobiernos para exigir mejoras en la enseñanza y el aprendizaje estudiantil -los padres de familia, los empresarios y la sociedad en general- en muchos casos quedan al margen del debate (FUSADES, 2018).

Generar mayor conocimiento y recomendaciones de política pública sobre la profesión docente y su formación deberá ser por tanto uno de los temas prioritarios en la agenda de desarrollo educativo de El Salvador, a fin de elevar la calidad de los aprendizajes, centrando la atención en cómo fortalecer la formación de los maestros 
y su labor en las aulas. Adicionalmente, hacer realidad una educación de calidad para todos, pasa necesariamente por el mejoramiento de la inversión en educación, y por el buen y acertado uso que se haga de los recursos.

Las transformaciones de los sistemas educativos para alcanzar mejores niveles de eficacia demandan esfuerzos sostenidos en períodos largos de tiempo. Por ello es importante establecer puntos de consenso entre los diferentes actores sociales, a fin de contar con políticas de largo plazo que permitan enfrentar los grandes desafíos de la educación: i) ampliación de la cobertura educativa, especialmente en parvularia y secundaria; ii) aumentar la equidad en el acceso a la educación; iii) elevar la eficiencia y la calidad de la educación en todos los niveles; iv) crear y mantener escuelas seguras; y v) promover la eficiencia y la sostenibilidad de la inversión en educación (FUSADES, 2011). Esto, de acuerdo con la evidencia internacional, constituye un factor predictivo de competitividad económica para los países.

\section{Doing Business}

Otro elemento de análisis en las causales de competitividad y crecimiento de la economía salvadoreña es la clasificación de país en el llamado "doing business" o facilidad para hacer negocios, que ubicó en 2016 a El Salvador en la posición 86 entre 189 países en el mundo (Grupo Banco Mundial, 2016), posición que denota algunas dificultades para hacer negocios y para la inversión de capital, que en el caso de El Salvador alcanzó en 2016 el 15\%, muy por debajo del promedio de América Latina que para ese año fue del 25\% (FMI, 2016).
$\mathrm{E}$ l informe Doing Business es una publicación insignia del Grupo Banco Mundial, que anualmente miden las regulaciones que favorecen o restringen la actividad empresarial, a partir de indicadores cuantitativos sobre las regulaciones empresariales y la protección de los derechos de propiedad privada que son comparables en 190 economías del mundo. Los informes analizan las regulaciones que afectan 11 áreas del ciclo de vida de una empresa. Diez de estas áreas se incluyen en la clasificación sobre la facilidad para hacer negocios: i) apertura de una empresa; ii) manejo de permisos de construcción; iii) obtención de electricidad; iv) registro de propiedades; v) obtención de crédito; vi) protección de los inversionistas minoritarios; vii) pago de impuestos; viii) comercio transfronterizo; ix) cumplimiento de contratos; $\mathrm{y}$ x) resolución de insolvencia. Doing Business también mide la regulación del mercado laboral, que no fue incluida en la medición del año 2018.

Doing Business 2018 (Reformando para la Creación de Empleos) reveló que en el año 2017 119 economías observaron mejoras en los marcos regulatorios locales. El informe, que comparó 190 economías en todo el mundo, identificó 264 reformas empresariales encaminadas a reducir la complejidad y el costo de cumplir con la legislación de los países, entre ellas las reformas en el área de apertura de empresas y en la obtención de créditos.

El Salvador, junto a otros 9 países (Brunei Darussalam, Tailandia, Malawi, Kosovo, India, Uzbekistán, Zambia, Nigeria, Djibouti) se encuentra entre las economías que mejoraron más en el periodo 2016/2017 en las áreas evaluadas por Doing Business, lo que significa 
que El Salvador está entre los 10 países con mayores mejorías en la facilidad para hacer negocios (Grupo Banco Mundial, 2018).

Muchos países en todos los continentes y regiones están implementando reformas que facilitan hacer negocios. Europa y Asia Central continúa siendo las regiones con la mayor proporción de economías donde se ha implementado al menos una reforma, seguidos de Asia del Sur y África Subsahariana.

En la clasificación global sobre la facilidad de hacer negocios, Doing Business 2018, es Nueva
Zelandia la que ocupa la primera posición en el ranking mundial, seguido en su orden por Singapur, Dinamarca, República de Corea, Hong Kong RAE, Estados Unidos, Reino Unido y Noruega. El Salvador gracias a los avances logrados en los últimos años, lo que le ha valido colocarse entre los primeros 10 países que más progreso han experimentado a nivel mundial, en el informe Doing Business de 2018, se ubica en la posición 73, solo por debajo de México, Chile, Perú, Colombia y Costa Rica a nivel latinoamericano. Esto significa un ascenso de 13 posiciones con respecto al informe de 2016 (Grupo Banco Mundial, 2018).

Tabla n..$^{\circ} 5$

Posiciones en Doing Business 2018

\begin{tabular}{c|c|c}
\hline Posición & Economía & Score \\
\hline 1 & Nueva Zelanda & 86.55 \\
\hline 2 & Singapur & 84.57 \\
\hline 3 & Dinamarca & 84.06 \\
\hline 4 & República de Corea & 83.92 \\
\hline 5 & Hong Kong SAR & 83.44 \\
\hline 6 & Estados Unidos & 82.54 \\
\hline 7 & Reino Unido & 82.22 \\
\hline 8 & Noruega & 82.16 \\
\hline 49 & México & 72.27 \\
\hline 55 & Chile & 71.22 \\
\hline 58 & Perú & 69.45 \\
\hline 59 & Colombia & 69.41 \\
\hline 61 & Costa Rica & 69.13 \\
\hline 73 & El Salvador & 66.42 \\
\hline 79 & Panamá & 65.27 \\
\hline 94 & Uruguay & 61.99 \\
\hline 97 & Guatemala & 61.18 \\
\hline
\end{tabular}

Fuente: Foro Económico Mundial, 2018 
La puntuación doing business de El Salvador en el informe de 2016 fue 62.76, pasando en el 2018 a 66.42, lo que revela un avance de 3.66 puntos. Esta puntuación refleja el desempeño de la economía en una escala de 0 a 100 , en donde 0 representa el rendimiento más bajo y 100 representa el desempeño más alto.

Al evaluar por áreas el desempeño de la economía salvadoreña se advierte que los avances más importantes en la facilidad para hacer negocios en los últimos tres años ha sido en el rubro pago de impuestos, con un crecimiento de 26.48 puntos, seguido de los rubros manejo de permisos de construcción (mejora de 5.14 puntos) y obtención de electricidad (mejora de 3.12 puntos). El resto de áreas que mide doing businnes se ha mantenido relativamente constante, pero sin decrecimiento. Ver Tabla n. ${ }^{\circ} 6$.

No obstante estos avances, debe decirse que la brecha de oportunidad de mejora en este indicador de desempeño de la economía salvadoreña es aún importante. Si bien la puntuación de país (66.42 en 2018) está por arriba del promedio latinoamericano (58.66 puntos), se advierte estancamiento y algunas dificultades para la apertura de negocios, registro de propiedades, obtención de créditos, protección de inversionistas minoritarios, cumplimiento de contratos y resolución de la insolvencia, este último con un leve decrecimiento en la puntuación. Para superar este estancamiento deberá promoverse el diseño una política pública de fomento de la investigación, desarrollo e innovación en El Salvador a fin de generar innovaciones y tecnologías que aumenten la productividad empresarial y por ende la competitividad del país frente a los competidores globales.

Los resultados de los informes doing businees al igual que los del ICG- son concordantes con los hallazgos del Programa para la Evaluación Internacional de Alumnos (PISA). Los países con mejor ranking en doing business, en la mayoría de los casos, lo son también en el ranking de PISA, lo que nuevamente advierte la alta correlación entre educación y competitividad de las economías, en este caso particular, con la facilidad para hacer negocios. Los ocho países con mejor ranking en doing business del período 2016-2018, se encuentran también entre los primeros 30 países con mejores resultados en las pruebas PISA del reporte de 2016. El ranking de PISA 2016, como se señaló antes, ubica a Singapur a la cabeza, seguido de Japón, Estonia, Taiwán, Finlandia, Macao, Canadá y Vietnam (OCDE, 2016). Ver Tabla n. ${ }^{\circ} 7$.

\section{Perspectivas y recomendaciones desde las políticas públicas en el sector Educación}

El Salvador continúa arrastrando un crecimiento lento y significativamente inferior al de buena parte de los países de la región latinoamericana, caracterizado por un contexto de escasa inversión, emigración elevada y forzada, déficit fiscal, alto endeudamiento y baja competitividad en general, a lo que se suma un entorno nacional de violencia, inseguridad e inviabilidad política. De allí que la reactivación de la economía salvadoreña y su inserción competitiva en la economía global requiera de medidas de diversa índole que aborden la multicausalidad del fenómeno. 


\section{Tabla n. ${ }^{\circ} 6$}

Desempeño de El Salvador en Doing Business, 2018

(Desagregado por áreas evaluadas)

\begin{tabular}{l|c|c}
\hline \multicolumn{1}{c|}{ Áreas evaluadas } & Score & Posición \\
\hline Doing business & $\mathbf{6 6 . 4 2}$ & $\mathbf{7 3}$ \\
\hline Apertura de una empresa & 78.88 & 139 \\
\hline Manejo de permisos de construcción & 60.16 & 88 \\
\hline Obtención de electricidad & 71.40 & 69 \\
\hline Registro de propiedades & 67.92 & 20 \\
\hline Obtención de crédito & 80 & 160 \\
\hline Protección de los inversionistas minoritarios & 38.33 & 61 \\
\hline Pago de impuestos & 77.35 & 43 \\
\hline Comercio transfronterizo & 89.29 & 105 \\
\hline Cumplimiento de contratos & 55.20 & 84 \\
\hline Resolución de insolvencia & 45.69 & \\
\hline
\end{tabular}

Fuente: Grupo Banco Mundial, 2018.

Tabla n. ${ }^{\circ} 7$

Comparación de ranking Doing Business 2018 y ranking PISA, 2016

\begin{tabular}{c|c|c}
\hline Economías & Ranking DB 2018 & Ranking PISA 2016 \\
\hline Nueva Zelanda & 1 & 12 \\
\hline Singapur & 2 & 11 \\
\hline Dinamarca & 3 & 9 \\
\hline República de Corea & 4 & 25 \\
\hline Hong Kong SAR & 5 & 15 \\
\hline Estados Unidos & 6 & 24 \\
\hline Reino Unido & 7 & 44 \\
\hline Noruega & 8 & 55 \\
\hline México & 49 & 58 \\
\hline Chile & 55 & 57 \\
\hline Perú & 58 & 47 \\
\hline Colombia & 59 & No participa \\
\hline Costa Rica & 61 & 47 \\
\hline El Salvador & 73 & \\
\hline Uruguay & 79 & 24 \\
\hline Po Banco & 2016 & 15 \\
\hline
\end{tabular}

Fuente: Grupo Banco Mundial, 2018.y OCDE, 2016. 
La inseguridad y violencia crónicas que vive E1 Salvador es una barrera para la competitividad y el desempeño económico del país. A 25 años de la firma de los acuerdos de paz, puede decirse que El Salvador está enfrentando una "nueva guerra" entre el Estado y las pandillas, cuya solución también se ve afectada por el clima de confrontación y polarización política que ha impregnado las estructuras de poder y que impide la toma de acuerdos para el desarrollo sostenible del país.

La apuesta a la educación, es sin duda una apuesta a la competitividad internacional, una apuesta a la inserción al nuevo escenario global, en donde la plataforma educativa de la población y su capacidad para generar valor en los procesos productivos, es la clave para la prosperidad económica. En tal sentido, deben hacerse los esfuerzos necesarios por asegurar una educación de calidad a todos los salvadoreños, no importando su condición socio económica. Las políticas de largo plazo en educación deberán complementarse con otras medidas de política pública orientadas a mejorar los contextos sociales y económicos de las familias, pues hay evidencia de que ello es un factor importante en los aprendizajes que puedan alcanzar los estudiantes.

Intentando correlacionar -y explicar también- la baja competitividad de la economía salvadoreña desde las intervenciones en Educación, se plantean las reflexiones y recomendaciones siguientes:

- Históricamente la política económica y la política educativa se han entendido como procesos separados que tienen lugar en la esfera del Estado y que se materializan mediante intervenciones (programas y acciones) en el ámbito económico y en el educativo de manera aislada. Las necesidades de competitividad y de inserción del país en los mercados globales demanda superar ese enfoque tradicional, por un enfoque articulador e integrador, en donde ambas esferas se retroalimentan mutuamente.

- A nivel global y de región latinoamericana existe una alta correlación entre avances significativos en educación y competitividad de las economías. Esto queda claro al comparar los rankings del Índice de Competitividad Global (ICG) y Doing Business con los resultados de PISA. Los países con mejores resultados de competitividad económica y facilidad para hacer negocios, están entre los primeros treinta países con mejores resultados de aprendizaje de sus estudiantes en las pruebas de PISA.

- A partir de la consideración anterior, cobra relevancia el alcance de acuerdos nacionales en el campo de la educación, a fin de establecer planes de desarrollo de largo plazo para una educación de calidad que promueva la formación de capacidades ciudadanas y productivas, para una inserción exitosa de la persona en la sociedad y en el mundo productivo, este último con orientación a los mercados internacionales.

- No es resultado de la casualidad que los países con alto índice de competitividad de sus economías -y desarrollo humano tambiénhayan logrado avances importantes en educación. Ello es el resultado a un acuerdo social que ha dado la prioridad debida a la salud, la educación, la capacitación, la ciencia y la tecnología; así como a la búsqueda 
permanente de apuestas económicas que les ha permitido mejorar progresivamente los indicadores de empleo e ingresos.

- Concebidas las políticas públicas como la herramienta para intervenir en los asuntos de interés colectivo, en la búsqueda de un acuerdo nacional en educación, deberá reconocerse la experiencia y éxito logrado por otros actores sociales distintos al Estado, llámense éstos iglesias, ONGs, municipalidades, comunidades locales y sociedad civil en general. Este buen trabajo debe ser reconocido y apoyado mediante estímulos financieros y una estratégica descentralización de responsabilidades. Asimismo se deberá invitar e integrar a este esfuerzo a otros actores sociales no tradicionales: empresarios, centros de trabajo, sindicaos, cooperativas, entre otros.

- Mejorar la competitividad de la economía salvadoreña, requiere mejorar la inversión en educación, así como ser más eficiente en el gasto público destinado a este sector, de manera que los limitados recursos se empleen estratégicamente en aquellos temas de relevancia educativa y mejora sustantiva de la educación nacional. En este sentido deberán eliminarse gastos superfluos en temas que abonan poco o nada a la calidad educativa. E1 gasto público en educación deberá focalizarse en temas clave, tales como: el mejoramiento de las competencias académicas y pedagógicas de los docentes, el fortalecimiento del rol y liderazgo de los directores de los centros educativos, el otorgamiento de mayores niveles de autonomía al centro escolar en los aspectos pedagógico, administrativo $\mathrm{y}$ financiero, así como en el fortalecimiento de la cultura de rendición de cuentas del centro escolar a sus comunidades educativas, mejoras en el currículo nacional de manera que se ajuste a las necesidades de formación en competencias productivas y ciudadanas para una mejor inserción de la persona a la sociedad y al mundo productivo, entre otros temas clave y estratégicos para el desarrollo educativo nacional.

- Incorporar al currículo nacional -desde la educación inicial y primaria- el desarrollo de capacidades para la innovación y el emprendedurismo, a fin de que en la edad productiva las personas tengan mejores condiciones para aportar valor en los procesos productivos, y en consecuencia, contribuir a la competitividad del país a nivel global.

- Siempre en la línea de hacer más eficiente la inversión, se debe efectuar un análisis de la cadena de valor del sector educación a fin de establecer los factores clave de éxito en los que se debe focalizar la inversión en educación, a fin de asegurar la mejor tasa retorno, y en consecuencia, el mayor valor agregado en el sector y con ello contribuir a una educación de mejor calidad y más pertinente a las necesidades de desarrollo económico y social del país.

- Contar con maestros bien formados y calificados es uno de los atributos más importantes que deben tener las escuelas para producir los mejores resultados (Bruns \& Luque, 2014; Hanushek, 2011; Hanushek \& Rivkin, 2006), de manera que es importante que los esfuerzos financieros y técnicos den la debida atención a la formación y 
profesionalización de los docentes, pues su contribución en la cadena de valor del sistema educativo es fundamental.

- Generar mayor conocimiento y recomendaciones de política pública sobre la profesión docente y su formación deberá ser por tanto uno de los temas prioritarios en la agenda de desarrollo educativo de El Salvador, a fin de elevar la calidad de los aprendizajes, centrando la atención en cómo fortalecer la formación de los maestros y su labor en las aulas.

- Por otra parte, si bien el tema de innovación, ciencia y tecnología se ha venido posicionando en la agenda de país en los últimos años, lo cierto es que los esfuerzos no han tenido la continuidad ni los recursos suficientes, esto debido a los vaivenes políticos provocados principalmente por los cambios de gobierno y la ausencia de acuerdos de nación en esta materia. Prevalece un discurso público triunfalista que celebra logros poco relevantes y poco asociados a asuntos de valor, que responde principalmente a un afán de clientelismo político.

Puede afirmarse que la capacidad de innovación de El Salvador es sumamente limitada, muy por debajo de los niveles mundiales y de la región latinoamericana, lo cual impone al país una barrera estructural para el crecimiento y competitividad de su economía. Como lo afirma la OCDE, la innovación es la ventaja comparativa de la economía del conocimiento, el motor más resistente a las crisis.

Desde las políticas públicas se debe promover la creación de sistemas nacionales de innovación, integrando a las diferentes instancias gubernamentales, a la empresa privada, al sector financiero, a las universidades, a la sociedad civil y a la cooperación internacional. La construcción de dichos sistemas requiere crear una serie de condiciones e incentivos fundamentales que aborden las causas estructurales del rezago: el fortalecimiento institucional, el mejoramiento de la infraestructura, la apuesta decidida al desarrollo del capital humano, un mejor marco regulatorio que fomente la participación de las empresas en la innovación; el fomento de programas de apoyo a la innovación y a las PYMES y en general una población bien educada, con capacidades productivas y ciudadanas puestas al servicio de los grandes objetivos del país.

\section{Bibliografía}

Bruns, B. and Luque, J. (2014). Great teachers. How to raise student learning in Latin America and the Caribbean, The World Bank, Washington DC.

FUSADES. (2011). Insumos para una Politica de Estado en Educación (Estudios Estratégicos - Competitividad para el Desarrollo 2011, San Salvador, E1 Salvador.

Organización para la Cooperación y el Desarrollo Económico (OCDE). (2016). Informe de PISA 2016. París, Francia: Autor.

MINED. (2017). Documento informativo para directores, docentes y estudiantes de educación media. Recuperado de www.mined.gob.sv

Schwab, K. (2015). The Global Competitiveness Report 2015-2016. World Economic Forum, Columbia University, USA. 
Schwab, K. (2016). The Global Competitiveness Report 2016-2017. World Economic Forum, Columbia University, USA.

Schwab, K. (2017). The Global Competitiveness Report 2017-2018. World Economic Forum, Columbia University, USA.
TIMSS. (2007). Resultados de El Salvador en Estudio TIMSS 2007. A Bridge School Improvement.

World Bank Group. (2018). Doing Business 2017. Equal Opportunity for All. A World Bank Group Flagship Report. 14th Edition, Washington DC. 